\title{
Chapter 14 \\ Rice Plant Biomineralization: Electron \\ Microscopic Study on Plant Opals \\ and Exploration of Organic Matrices \\ Involved in Biosilica Formation
}

\author{
Noriaki Ozaki, Takuya Ishida, Akiyoshi Osawa, Yumi Sasaki, Hiromi Sato, \\ Michio Suzuki, Keiju Okano, and Yuko Yoshizawa
}

\begin{abstract}
Biologically formed amorphous silica (biosilica) is widely found in diatoms, marine sponges, terrestrial plants, and bacteria, some of which have been well characterized. Although rice plants produce large amounts of biosilica (plant opal) in their leaf blades and rice husks, the molecular mechanism of biomineralization is still poorly understood. In the present study, we investigated the fundamental properties of plant opal in leaf blades of the rice plants (Oryza sativa) by scanning electron microscopy (SEM) equipped with energy-dispersive X-ray spectroscopy. The number of fan-shaped plant opal increases in the motor cells (bubble-shaped epidermal cells) during heading time. High-resolution SEM analysis revealed that the plant opals are composed of nanoparticles, as is the case with diatom silica and siliceous spicule of sponge. Organic matrices in biominerals have been considered to control mineralization. Biosilicas in diatom and marine sponge are formed under ambient conditions using organic matrices, unique proteins, and long-chain polyamines. In this study, we report the establishment of purification method of plant opals from rice leaf blades. Finally, we succeeded in extracting organic matrices from the purified plant opal.
\end{abstract}

Keywords Biomineralization $\cdot$ Biosilica $\cdot$ Organic matrix $\cdot$ Plant opal $\cdot$ Rice plant

\footnotetext{
N. Ozaki $(\square) \cdot$ T. Ishida $\cdot$ A. Osawa $\cdot$ Y. Sasaki $\cdot$ H. Sato $\cdot$ K. Okano $\cdot$ Y. Yoshizawa Department of Biotechnology, Faculty of Bioresource Sciences, Akita Prefectural University, Akita, Japan e-mail: ozanor@akita-pu.ac.jp; m14g005@akita-pu.ac.jp; keijuo@ akita-pu.ac.jp; yyoshizawak@akita-pu.ac.jp

M. Suzuki

Department of Applied Biological Chemistry, Graduate School of Agricultural and Life Sciences, The University of Tokyo, Tokyo, Japan

e-mail: amichiwo@mail.ecc.u-tokyo.ac.jp
} 


\subsection{Introduction}

Biomineralization is widespread phenomenon by which organisms produce minerals by using organic matrices under ambient conditions (Lowenstam and Weiner 1989). The resulting minerals, termed biominerals, have a specific morphology and demonstrate excellent physical properties. Biogenic amorphous silica (biosilica) is known as one of the representative biominerals. Biosilica is widely observed in skeleton of diatoms, spicules of marine sponges, spore coats of bacteria, and epicuticles of certain higher plants. As in the case of other biominerals, organic matrices in biosilicas are thought to be associated with silica formation. Until now, in diatoms, glass sponges, and certain facultative bacteria, several organic matrices involved in silica formation have previously been identified, such as unique proteins (silaffin, glassin, and CotB1) and long-chain polyamines (LCPAs) (Sumper and Kröger 2004; Shimizu et al. 2015; Matsunaga et al. 2007; Motomura et al. 2016). These matrices are highly charged and have been shown to promote silica formation from monosilicic acid solution near a neutral $\mathrm{pH}$. On the other hand, there are few studies on silica formation of higher plants. The best-known example of silicon accumulating plants, rice plants (Oryza sativa), produces a large amount of biosilicas (plant opal) in their leaf blades and rice husks. Silicon uptake mechanism from soil is transporter mediated and energy dependent (Ma et al. 2006). Plant opal deposition has been shown to improve disease resistance, light interception, and mechanical properties (Ma and Takahashi 2002). Despite the importance of plant opal in rice plants, information on the molecular mechanisms involved in plant opal formation is very limited. To date, there are no published reports on organic matrices from plant opal of rice, as far as we know. In the present work, we have investigated the fundamental properties of plant opals by microscopic analyses and extracted an organic matrix from fan-shaped plant opals.

\subsection{Materials and Methods}

\subsubsection{Plant Materials and Microscopy}

The leaf blades of rice plants (Oryza sativa cv. Akita-sake-komachi) were collected from paddy field in Akita Prefectural University. Optical microscope (BX51, Olympus) and field emission SEM (SU-8010, Hitachi) were used to analyze the microstructure of leaf blades and morphology of plant opals. The chemical composition of silica was confirmed with an energy-dispersive X-ray spectroscope (EDX; EMAX x-act, HORIBA). Prior to counting the number of fan-shaped opal, the leaf blade was incinerated at $550{ }^{\circ} \mathrm{C}$. The ashed sample was carefully placed on Superfrost micro slides (Matsunami) and observed with the optical microscope. 


\subsubsection{Extraction of Organic Matrices from Plant Opals}

Plant opals were separated from mature leaves according to the method of Setoguchi et al. (1990) with slight modifications. After washing with distilled water, rice leaf blades were cut into small pieces and ground with a mixer mill. The homogenate was passed through a nylon mesh filter of $258 \mu \mathrm{m}$ pore size (NB60, Atflon). The filtrate containing plant opals is put on a watch glass, and heavier fan-shaped plant opals were separated from lighter small leaf fragment by a series of decantation. Cell walls bound to fan-shaped plant opals were removed by sulfuric acid and cellulase (Onozuka R-10, Wako, Osaka) treatments. The resulting fan-shaped opals were suspended in $4 \mathrm{M}$ hydrogen fluoride (HF) solution and left for $2 \mathrm{~h}$ at room temperature. After centrifugation for $10 \mathrm{~min}$ at $2000 \mathrm{~g}$, the supernatant was subjected to dialysis (Float-A-Lyzer, Spectra-Por) against distilled water. The dialysate (HF-soluble fraction) was lyophilized and the resulting organic matrices were subjected to tricine-SDS-PAGE. Organic matrices were detected by Coomassie Brilliant blue (CBB; EzStain AQua, ATTO) and silver (SilverXpress, Thermo Fisher) staining. The HF-soluble fraction passed through the $30 \mathrm{kDa}$ molecular weight cutoff filter (Amicon Ultra 15, Millipore) was used to raise antibody in rabbits through a commercial source. Another set of sample was subjected to SDS-PAGE and subsequent blotting to a PVDF membrane. The membrane was blocked with 5\% skim milk diluted in TBS (Tris-buffered saline; $50 \mathrm{mM}$ Tris, $0.85 \% \mathrm{NaCl}, \mathrm{pH} 7.2$ ) with Tween $20(0.05 \%)$ for $1 \mathrm{~h}$ and then incubated in the primary antibody solution $(1: 100)$ for $1 \mathrm{~h}$ at room temperature. After washing, it was incubated in 1:3000 diluted solution of AP-conjugated goat anti-rabbit IgG secondary antibody (Bio$\mathrm{Rad}$ ) for $1 \mathrm{~h}$ at room temperature. The target protein was visualized using a BCIP/ NBT substrate system (Bio-Rad).

\subsection{Results and Discussion}

\subsubsection{Morphology and Function of Plant Opals}

Representative SEM micrographs of several types of plant opal from rice leaf blades are shown in Fig. 14.1. SEM-EDX analysis proved that all types of plant opals were composed of silicon, oxygen, and carbon (data not shown). The surface of leaf blades consists of silicified cells, called long cells making plate-shaped opals (Fig. 14.1a), prickle hair (Fig. 14.1b), and short cells making dumbbell-shaped opals (Fig. 14.1c). Our preliminary study revealed that plant opals on leaf surface are formed and silicified within 2 weeks after germination (data not shown). The possible function of these opals on the leaf surface is considered to protect against biotic and abiotic stress, such as pathogenic bacteria and dryness. Only the 

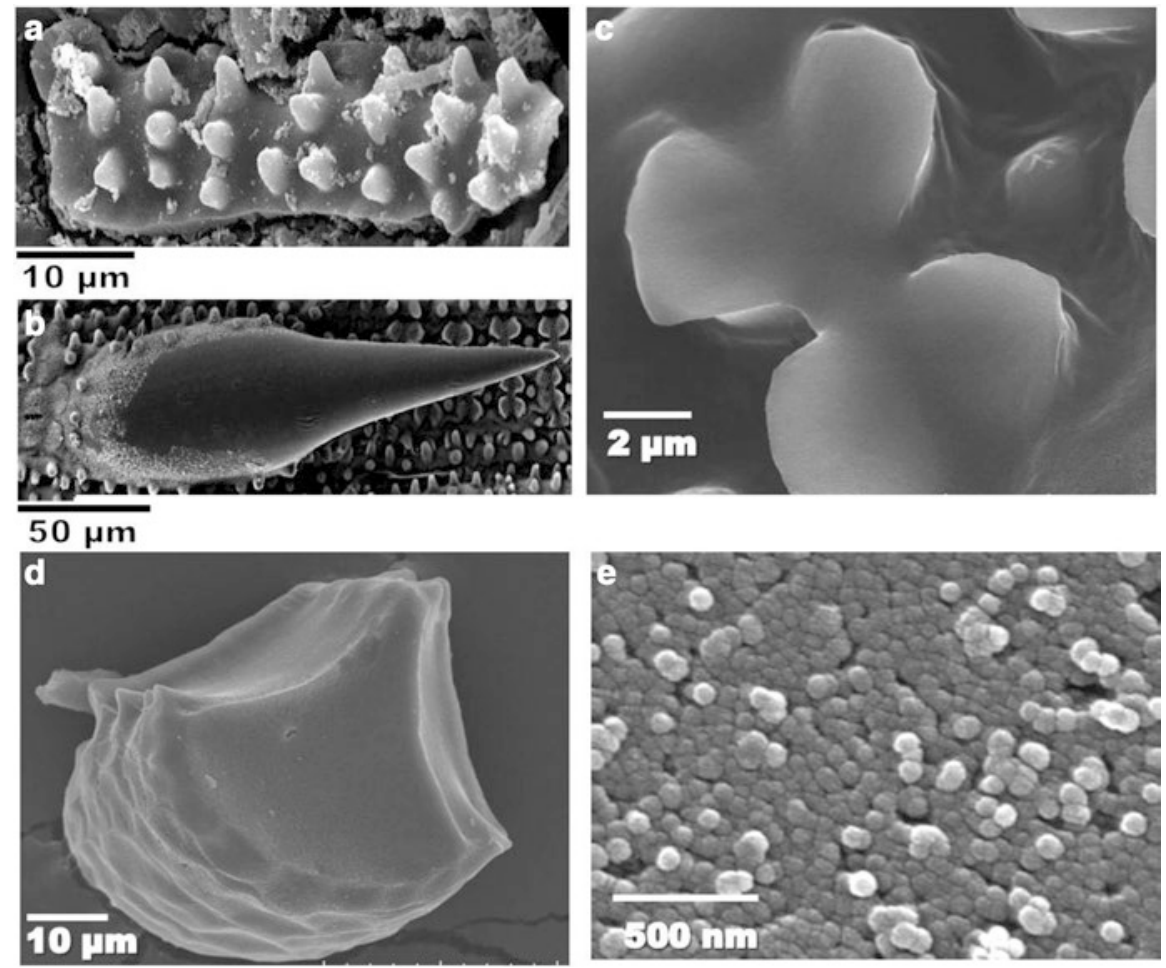

Fig. 14.1 Representative scanning electron microscope (SEM) micrographs of plant opals in rice leaf blades. (a) Plate-shaped opal. (b) Silicified prickle hair. (c) Dumbbell-shaped opals. (d) Purified fan-shaped opal. (e) Silica nanoparticles constituting fan-shaped opal

fan-shaped plant opal (Fig. 14.1d) is formed inside the leaf blade. Our research group confirmed that fan-shaped opal contains highest concentration of silica (Sato et al. 2017). Based on higher magnification image of fan-shaped opals, we found that the opal comprised fine particles with several tens of nanometer in diameter (Fig. 14.1e). Similar silica nanoparticles are also found in the spicules of marine sponges (Aizenberg et al. 2005)

In order to investigate the formation period of the fan-shaped opal, we collected rice leaves from July to October. To count the number of opals, each collected leaf blades were treated by calcination at $550{ }^{\circ} \mathrm{C}$. The optical micrograph of surface of leaf blade after calcination is shown in Fig. 14.2a. Fan-shaped plant opals (arrowheads) were observed in the motor cells between the veins of the leaf (white dotted line) and were aligned like a backbone. As shown in Fig. 14.2b, the number of fan-shaped opals sharply increased around the heading time. The heading time (dotted line) in Akita prefecture was early August with strong sunlight. Although the function of the fan-shaped opal had not been clarified (Kaufman et al. 1979; 

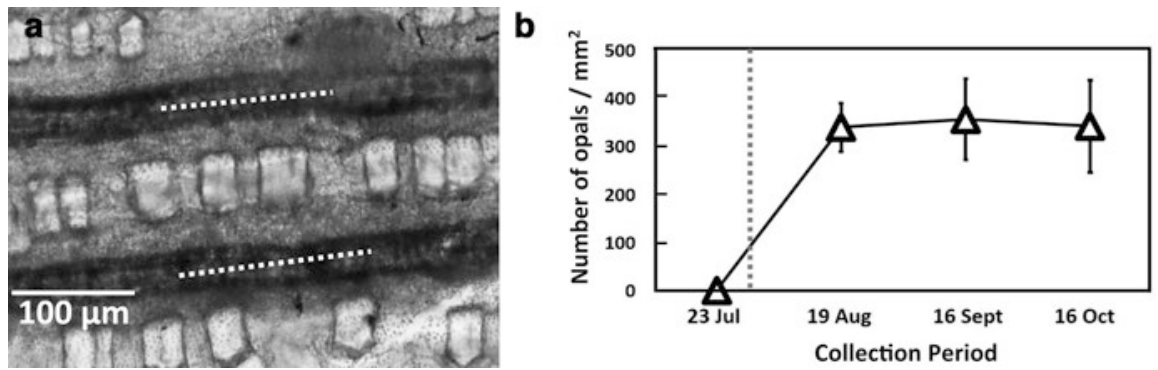

Fig. 14.2 Fan-shaped plant opal formation in the flag leaf of rice. (a) Optical micrograph of surface of ashed leaf blade. White dotted lines indicate vascular bundle. When looking at fan-shaped opals from another angle, it looks like rectangle structures (arrowheads). (b) Time course of formation of fan-shaped plant opals. Dotted line indicates the heading time (ear-forming period). The number of fan-shaped opal was measured by counting rectangle structures (arrowhead in a)

Agarie et al. 1996), we suggested the possibility that fan-shaped opals have a role in guiding light to chloroplast by optical simulation and actual optical experiment (Sato et al. 2016). Another possibility is that the fan-shaped opals formed inside the leaf play a role like a bone and improve the posture for light interception ( $\mathrm{Ma}$ and Takahashi 2002). In either case, the fan-shaped opal may promote photosynthesis necessary for panicle formation.

\subsubsection{Organic Matrices from Separated Plant Opals}

Although organic matrices from biosilica are considered to play important roles in silica formation, there are few reports about organic compounds in plant opal (phytolith) of higher plants (Elbaum et al. 2009). It was difficult to extract organic matrices from opals, due to complex structure consisting of cuticle and silica layer. So, we developed a novel silica purification method and succeeded in extracting organic matrices from the separated plant opal. Cell walls bound to the silica were decomposed by successive treatments with sulfuric acid and cellulase. The HF-soluble fraction from opals was subjected to tricine-SDS-PAGE. At least two main bands (arrowheads) were detected using CBB staining (Fig. 14.3a). On the other hand, only one band was stained with silver staining (Fig. 14.3b, arrowhead). From these results, the band with an apparent molecular mass of $10 \mathrm{kDa}$ is referred to as the HF-soluble protein. Only the $10 \mathrm{kDa}$ protein strongly reacted with the antibody raised against the fraction containing HF-soluble protein (Fig. 14.3c, arrowhead), indicating that the antibody was highly specific to this protein. The amino acid sequence analysis by LC-MS/MS and immunohistochemical analysis of this protein are in progress. Detailed results will be reported elsewhere. 


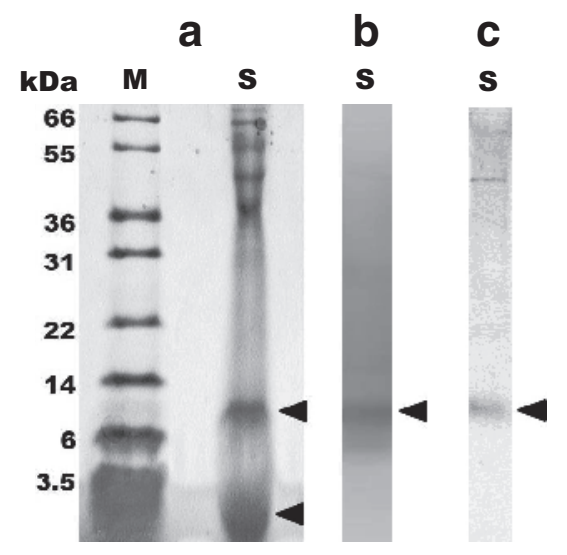

Fig. 14.3 Tricine-SDS-PAGE (12.5\%) and Western blot analyses of the HF-soluble organic matrices extracted from fan-shaped plant opals. (a) Two major bands (arrowheads) were detected by CBB staining. (b) Only one band (arrowhead) was positive for silver staining. (c) Western blotting with an anti-HF-soluble proteins antiserum. Only one immunoreactive band was detected at about $10 \mathrm{kDa}$. $M$ molecular mass standards. $S$ HF-soluble fraction

\section{References}

Agarie S, Agata W, Uchida H, Kubota F, Kaufman PB (1996) Function of silica bodies in the epidermal system of rice (Oryza sativa L.): testing the window hypothesis. J Exp Bot 47:655-660

Aizenberg J, Weaver JC, Thanawala MS, Sundar VC, Morse DE, Fratzl P (2005) Skelton of Euplectella sp: structural hierarchy from nanoscale to the macroscale. Science 309:275-278

Elbaum R, Melamed-Bessudo C, Tuross N, Levy A, Winer S (2009) New methods to isolate organic materials from silicified phytolith reveal fragmented glycoproteins but no DNA. Quat Int 193:11-19

Kaufman PB, Takeoka Y, Carlson TJ, Bigelow WC, Jones JD, Morre PH, Ghosheh NS (1979) Studies on silica deposition in sugarcane, using scanning electron microscopy, energy dispersive X-ray analysis, neutron activation analysis, and light microscopy. Phytomorphology 29:185-193

Lowenstam HA, Weiner S (1989) On biomineralization. Oxford University Press, London

Ma JF, Takahashi E (2002) Functions of silicon in plant growth. In: Soil, fertilizer, and plant silicon research in Japan. Elsevier Science, Amsterdam

Ma JF, Tamai K, Yamaji N, Mitani N, Konishi S, Katsuhara M, Ishiguro M, Murata Y, Yano M (2006) A silicon transporter in rice. Nature 440:688-691

Matsunaga S, Sakai R, Jimbo M, Kamiya H (2007) Long-chain polyamines (LCPAs) from marine sponge: possible implication in spicule formation. Chem Biochem 8:1729-1735

Motomura K, Ikeda T, Matsuyama S, Mohamed A, Tanaka T, Ishida T, Hirota R, Kuroda A (2016) The C-terminal zwitterionic sequence of CotB1 is essential for biosilicification of the Bacillus cereus spore coat. J Bacteriol 198:276-282

Sato K, Yamauchi A, Ozaki N, Ishigure T, Oaki Y, Imai H (2016) Optical properties of biosilicas in rice plants. RSC Adv 6:109168-109173

Sato K, Ozaki N, Nakanishi K, Sugahara Y, Oaki Y, Salinas C, Herrera S, Kisailus D, Imai H (2017) Effects of nanostructured biosilica on rice plant mechanics. RSC Adv 7:13065-13071 
Setoguchi H, Okazaki M, Suga S (1990) Calcification in higher plants with special reference to cystoliths. In: Origin, evolution, and modern aspects of biomineralization in plants and animals. Plenum Press, New York

Shimizu K, Amano T, Bari MR, Weaver JC, Arima J, Mori N (2015) Glassin, a histidine-rich protein from the siliceous skeletal system of the marine sponge Euplectella, directs silica polycondensation. PNAS 112:11449-11454

Sumper M, Kröger N (2004) Silica formation in diatoms: the function of long-chain polyamines and silaffins. J Mater Chem 14:2059-2065

Open Access This chapter is licensed under the terms of the Creative Commons Attribution 4.0 International License (http://creativecommons.org/licenses/by/4.0/), which permits use, sharing, adaptation, distribution and reproduction in any medium or format, as long as you give appropriate credit to the original author(s) and the source, provide a link to the Creative Commons license and indicate if changes were made.

The images or other third party material in this chapter are included in the chapter's Creative Commons license, unless indicated otherwise in a credit line to the material. If material is not included in the chapter's Creative Commons license and your intended use is not permitted by statutory regulation or exceeds the permitted use, you will need to obtain permission directly from the copyright holder.

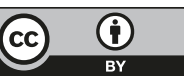

